\title{
Novel insights into transmission routes of Mycobacterium avium in pigs and possible implications for human health
}

\author{
Angelika Agdestein ${ }^{1}$, Ingrid Olsen', Anne Jørgensen², Berit Djønne ${ }^{1}$ and Tone B Johansen ${ }^{*}$
}

\begin{abstract}
Mycobacterium avium infection is a severe condition in humans, whereas pigs are often subclinically infected. Pig carcasses represent a possible source of human infection. Faecal excretion of M. avium was recently demonstrated in experimentally infected pigs, along with detection of $M$. avium in apparently normal lymph nodes. The present study investigates faecal excretion in naturally infected herds and the presence of live mycobacteria in lymph nodes. Two pig herds (A and B), with a history of sporadically suspected M. avium infection were sampled. Herd B used peat, as opposed to Herd A. Samples from peat, sawdust, drinking water, faeces and lymph nodes were collected. Identification of mycobacteria was performed by 165 rDNA sequencing and PCR. Mycobacterium avium isolates were analysed by Multi-Locus Variable Number of Tandem repeat Analysis (MLVA). Mycobacterium avium subsp. hominissuis was detected in samples of faeces, peat and lymph nodes from Herd B, often with identical MLVA profiles. Additionally, other non-tuberculous mycobacteria (NTM) were found in the same material. The absence of macroscopic lymph node lesions in the presence of $M$. avium subsp. hominissuis was frequently demonstrated. In Herd A, only one NTM isolate, which proved not to be M. avium, was found. Faeces might facilitate transmission of M. avium subsp. hominissuis between pigs and maintain the infection pressure in herds. The low incidence of macroscopic lesions together with the massive presence of $M$. avium subsp. hominissuis in lymph nodes from pigs kept on peat raises questions related to animal husbandry, food safety and human health.
\end{abstract}

\section{Introduction}

Non-tuberculous mycobacteria (NTM) comprise a wide group of bacteria, where many can act as opportunistic pathogens [1]. Mycobacterium avium subsp. hominissuis is the most commonly detected NTM causing infection in humans and pigs [2]. Mycobacterium avium subsp. avium occasionally infects pigs and humans, but is generally regarded as an obligate pathogen of birds, causing contagious avian tuberculosis [3]. Only $M$. avium subsp. hominissuis has been detected as the cause of porcine and human cases of M. avium infection in Norway [4]. However, data is limited in this regard. Less frequently, other NTM species are described in association with porcine lymphadenitis [5], and some of these have also been described as causative agents in human disease [6].

\footnotetext{
* Correspondence: tone.johansen@vetinst.no

${ }^{1}$ Norwegian Veterinary Institute, P.O. Box 750 Sentrum, N-0106 Oslo, Norway Full list of author information is available at the end of the article
}

In humans, pulmonary or systemic manifestations of M. avium infection are severe and mainly affect immunocompromised patients and patients with underlying chronic lung conditions [1]. These cases require long-term multi-drug treatment, with antibiotic resistance being a complicating factor. Another typical manifestation of $M$. avium infection is lymphadenitis in the head-and-neck region of children [7], a condition to be treated by surgical intervention. In pigs, no predisposition to severe clinical manifestations of M. avium infection has been described. Outbreaks of $M$. avium infection with marked clinical illness are relatively rare in pigs, but in the sporadically reported cases the infection seems to cause reproductive disorders $[8,9]$. The most common manifestation of infection in pigs, however, is characterised by subclinical infection of the lymph nodes and lymphatic tissues of the digestive tract, including the mandibular lymph nodes $[10,11]$. Dissemination to liver and lungs may also be detected at slaughter without any preceding 
pronounced clinical symptoms [12]. Inspection and incision of mandibular lymph nodes has been the routine examination for mycobacterial infection at abattoirs, but is no longer practiced in all Norwegian abattoirs. Infection with mycobacteria typically induces formation of granulomatous lesions [13], and the detection of these might cause partial or total condemnation of pig carcasses at the abattoir and lead to financial losses for the farmer.

Cases of human infection with $M$. avium have typically been traced to drinking water systems, saunas, pools and organic environmental materials [14-17]. However, studies demonstrating $M$. avium subsp. hominissuis in muscle tissue of experimentally infected pigs $[18,19]$ and in lymph nodes without visible lesions at slaughter [20,21], together with the close genetic relationship between isolates from humans and pigs [4,22-25], make it difficult to rule out the zoonotic potential of $M$. avium.

The most frequently used bedding materials in the swine industry are sawdust and peat. Peat has become increasingly popular in the swine industry the last years, both as an iron enriched feed supplement and as bedding. As bedding, peat has many advantages, like regulation of intestinal function, absorption of moisture and supporting the pigs' natural behavior. However, both peat and sawdust are well known sources for $M$. avium infection $[20,26,27]$. Czech screening studies of environmental samples from pig herds also detected $M$. avium subsp. hominissuis in water, pig faeces, and invertebrates [28]. A recent experimental infection study in Norwegian pigs compared one clinical M. avium subsp. hominissuis isolate from a pig and one clinical $M$. avium subsp. avium isolate from a bird. The study demonstrated faecal excretion of the $M$. avium subsp. hominissuis isolate exclusively [21]. The pronounced shedding was detected six weeks after infection, at about 12 weeks of age. However, in the same study, both subspecies showed similar potentials of establishing infection in pigs.

To the author's best knowledge, no studies have yet explored the role of faecal excretion of M. avium on transmission between pigs in problem herds. The aim of the present study was to investigate the presence of $M$. avium in herds with a history of sporadic cassations due to mycobacteriosis, by examining faeces and lymph nodes of the same group of animals, as well as environmental sources, and by characterising the genetic relationship between the isolates. Additionally, the study aims at assessing the presence of $M$. avium in macroscopically unapparent lymph nodes in natural infected herds, and to hereby elucidate aspects of food safety.

The presence of M. avium subsp. hominissuis was confirmed in one of the two herds, both in faeces, lymph nodes and in peat, in addition to other NTM species.
Multi-Locus Variable Number of Tandem repeat Analysis (MLVA) analysis of a selection of the M. avium subsp. hominissuis isolates identified 13 different profiles and demonstrated clustering of isolates from peat, faeces and lymph nodes. Also, the presence of $M$. avium subsp. hominissuis and other NTM was frequently demonstrated in a high proportion of lymph nodes of normal appearance at meat inspection. In contrast, $M$. avium was not detected in any of the samples from the other herd, and only one NTM isolate was detected in a faecal sample. This herd did not use peat as bedding for the animals.

\section{Materials and methods \\ Herds and animals}

Two commercial pig herds practicing combined breeding and fattening of grower pigs, further referred to as Herd A and B, were selected as the study population. Both herds were situated in south-eastern Norway, and had previous reported condemnation of carcasses at slaughter, due to macroscopic changes suggestive of mycobacterial infection in lymph nodes or other internal organs. In Herd A, M. avium infection had sporadically been confirmed by culture of tissue samples with lesions during the preceding three years. No such confirmation had been done in Herd B.

Herd A is an integrated farm with 135 breeding sows, weaning about 3500 piglets per year and producing about 2000 slaughter pigs per year. All pigs are kept indoors. Sows with piglets and weaned piglets are kept in different rooms. Dry sows are loose on sawdust bedding. Slaughter pigs are kept in ordinary pens in another house. Herd B is an integrated farm with 68 breeding sows, weaning about 1500 piglets per year and producing approximately 800 slaughter pigs per year. All pigs are kept indoors. Sows with piglets and weaned piglets are kept in the same room. Dry sows are loose on deep straw bedding and slaughter pigs are kept in ordinary pens. In Herd A, sawdust was used as bedding while in Herd B, peat and sawdust were routinely used as bedding for piglets and weaned pigs up to the age of nine weeks. Water was provided from the public drinking water system in both herds, and the animals were fed commercially produced feed (Felleskjøpet ${ }^{\circ}$, Oslo, Norway), which had been heat-treated and not added any meat products. No interventions were done concerning the regular everyday animal husbandry or feeding in the herds within the sampling period.

\section{Sampling}

Environmental samples were collected from sawdust, peat and drinking water used at the facilities (Table 1). Sampling of sawdust and peat was performed in storage rooms by random collection of $20 \times 10 \mathrm{~g}$ of each type of 


\begin{tabular}{lcc}
$\begin{array}{l}\text { Table } \mathbf{1} \text { Number of samples from pigs and their } \\
\text { environment examined for mycobacteria in two } \\
\text { commercial herds }\end{array}$ & \\
\hline Sample type & Herd A & Herd B \\
\hline Peat & na & 20 \\
Water & 1 & 1 \\
Sawdust & 20 & 20 \\
Faeces from sows & 15 & 17 \\
Faeces at 6 weeks of age & 95 & 146 \\
Faeces at 8 weeks of age & 95 & 164 \\
Mandibular lymph nodes & 95 & 123 \\
Jejunal lymph nodes & nd & 127 \\
\hline
\end{tabular}

na $=$ not applicable, nd $=$ not done.

material, whereas one sample of $10 \mathrm{~L}$ of drinking water was collected directly from an indoor water tap within each facility.

Individual faecal samples were collected rectally from the pigs at the age of six and eight weeks and from their mother sows. In Herd A, 95 grower pigs and 15 sows were sampled, and in Herd B, 146 and 164 pigs were sampled at weeks six and eight respectively, in addition to 17 sows (Table 1). One mandibular lymph node was collected from each animal at conventional slaughter (Table 1). Slaughter of the animals from Herd A was concentrated on the same day at the age of approximately 23 weeks, whereas the animals in Herd B were slaughtered in groups on a weekly basis, at the age of approximately $24,25,26$ and 28 weeks. In addition to the mandibular lymph nodes, one jejunal lymph node per animal was sampled from Herd B (Table 1). The selection of the jejunal lymph node samples was performed randomly in each animal, unless abnormal findings in consistency, size or colour were observed. Such altered lymph nodes were deliberately prioritized for sampling.

\section{Culturing, isolation and identification of mycobacteria}

Lymph nodes were incised and inspected prior to processing in the laboratory, and the presence of macroscopic lesions compatible with caseous necrosis and/or calcification was recorded. All samples were decontaminated as previously described [20] and cultured on slants of Middlebrook 7H10 (BD Diagnostics, Sparks, MD, USA) w/10\% oleic acid (BD Diagnostics), with and without antibiotics and fungicides (final concentrations of $100 \mu \mathrm{g} / \mathrm{mL}$ carbenicillin, $200 \mathrm{U} / \mathrm{mL}$ polymyxin B sulphate, $19.5 \mu \mathrm{g} / \mathrm{mL}$ trimethoprim lactate and $10 \mu \mathrm{g} / \mathrm{mL}$ amphotericin B), Dubos PS [29] and Stonebrink's medium (BD Diagnostics). Slants were incubated for eight weeks at $37{ }^{\circ} \mathrm{C}$, and colonies resembling mycobacteria were sub-cultured on Middlebrook 7H10 and the medium they were initially observed to grow on. On primary isolation, attempt was made to pick one colony of each morphotype, when more than one was present.

Isolates shown to be acid-fast rods by the Ziehl-Neelsen (ZN) staining method were further examined by PCR targeting the $M$. avium specific IS element IS1245 (primers p40 and p41) and the M. avium subsp. avium specific IS901 (primers p901a and p901c) as previously described [30,31]. Isolates negative on the PCR reactions were analysed further by $16 \mathrm{~S}$ rDNA sequencing of the $151 \mathrm{bp}$ hypervariable region A to identify species, as described [32]. Primers are presented in Additional file 1. Sequencing reactions were run partly in-house and partly by GATC Biotech (Konstanz, Germany), and the obtained sequences were analysed by using the Basic Local Alignment Search Tool (BLAST) [33] (National Center for Biotechnology Information (NCBI), Bethesda, MD). Maximum scores and maximum identity of $\geq 99 \%$ were accepted.

\section{Investigation of relatedness between $M$. avium isolates} Isolates of $M$. avium were analysed by Multi-Locus Variable Number of Tandem repeat Analysis (MLVA) of 8 loci, as previously described [34]. Loci and primers are presented in Additional file 1. The PCR was performed using HotStar Taq polymerase (Qiagen, Hilden, Germany) at the following thermocycling conditions: Initially $95^{\circ} \mathrm{C}$ for $15 \mathrm{~min}$, then 40 cycles of $30 \mathrm{~s}$ at $95^{\circ} \mathrm{C}, 30 \mathrm{~s}$ at $58{ }^{\circ} \mathrm{C}$, and $30 \mathrm{~s}$ at $72{ }^{\circ} \mathrm{C}$, and finally 1 cycle of $7 \mathrm{~min}$ at $72{ }^{\circ} \mathrm{C}$. The reference strain M. avium subsp. avium ATCC 25291 was used as positive control in each run. Sizes of the PCR products, reflecting differences in repeat numbers, were determined by using the Agilent Bioanalyzer ${ }^{\circ}$ (Agilent Technologies, Santa Clara, CA, USA) and converted to a corresponding number for each locus as described by Thibault et al. [34]. Cluster analysis was performed using the categorical method and the unrooted UPMGA tree in Bionumerics 6.1 (Applied Maths, Sint-MartensLatem, Belgium). Only isolates of $100 \%$ similarity, i.e. isolates having the same number of tandem repeat in each locus, were assigned to the same cluster.

\section{Results}

\section{Culturing, isolation and identification of mycobacteria}

No mycobacteria were detected in samples of sawdust and water from either of the two herds.

In Herd $\mathrm{A}$, only one mycobacterial isolate was detected. It was found in a faecal sample from a six week old pig, and identified as M. senuense. Neither macroscopic lesions nor mycobacteria were detected in mandibular lymph nodes from Herd A.

In Herd B, mycobacterial species were detected in peat, and in faeces from sows and piglets. Nineteen samples of peat $(95 \%)$, three samples of sow faeces $(18 \%)$, and 44 faecal samples (14\%) from six and eight week old 
pigs contained mycobacteria. Mycobacterium avium subsp. hominissuis was detected from 11 samples of peat (55\%) and from seven faecal samples (2\%) from six and eight week old pigs, while $M$. avium was not detected in sow faeces. A total of 16 isolates of $M$. avium subsp. hominissuis from the 11 positive peat samples and 11 isolates from the seven positive faecal samples were analysed by MLVA. Other mycobacterial species detected in peat samples were $M$. bohemicum (45\% of samples), M. malmoense (20\%), M. palustre (15\%) and M. celatum (5\%). Mycobacterium malmoense was identified in faecal samples from two sows, and M. celatum from one. In the 310 faecal samples collected from six and eight week old animals, M. malmoense (5\%), M. triviale (5\%) M. bohemicum (1\%), M. celatum (1\%) and M. branderi (0, 5\%) were detected. Numbers of isolates of each mycobacterial species within the volume of each sample type are shown in Table 2.

In the examined mandibular and jejunal lymph nodes from the animals in Herd B, mycobacterial species were detected in $69 \%$ and $48 \%$, respectively. Mycobacterium avium subsp. hominissuis was found in 81 mandibular (66\%) and in 60 jejunal lymph nodes (47\%) (Table 2). The detection frequency increased with the age of the slaughtered pigs at sampling, developing from $60 \%$ at 24 weeks to $80 \%$ at 28 weeks in mandibular lymph nodes, and from $33 \%$ to $72 \%$ in jejunal lymph nodes (Table 3). Other mycobacterial species cultured from the total amount of 250 lymph nodes were M. bohemicum (3\% of samples), M. celatum (3\%) and M. palustre (0.4\%). These NTM were detected alone or together with $M$. avium subsp. hominissuis in the same sample. Also, five additional isolates of acid fast rods remained unidentified, due to the quality of sequences being too poor for $16 \mathrm{~S}$ rDNA analysis.

Table 2 Number of samples from Herd B where mycobacterial species were detected

\begin{tabular}{|c|c|c|c|c|c|c|}
\hline \multirow{3}{*}{$\begin{array}{l}\text { Sample } \\
\text { material }\end{array}$} & \multirow[t]{2}{*}{ Peat } & \multicolumn{3}{|c|}{ Faeces } & \multicolumn{2}{|c|}{ Lymph nodes } \\
\hline & & Sows & $\begin{array}{l}16 \text { weeks } \\
\text { of age }\end{array}$ & $\begin{array}{l}8 \text { weeks } \\
\text { of age }\end{array}$ & Mandibular & Jejunal \\
\hline & 20 & 17 & 146 & 164 & 123 & 127 \\
\hline $\begin{array}{l}\text { M. avium subsp. } \\
\text { hominissuis }\end{array}$ & 11 & - & 2 & 5 & 81 & 60 \\
\hline M. branderi & - & - & 1 & - & - & - \\
\hline M. bohemicum & 9 & - & - & 4 & 8 & - \\
\hline M. celatum & 1 & 1 & - & 2 & 7 & 1 \\
\hline M. malmoense & 4 & 2 & 9 & 8 & - & - \\
\hline M. palustre & 3 & - & - & - & 1 & - \\
\hline M. triviale & - & - & 9 & 5 & - & - \\
\hline $\begin{array}{l}\text { M. spp. } \\
\text { (unidentified) }\end{array}$ & - & - & - & 3 & 2 & - \\
\hline
\end{tabular}

Table 3 Detection frequencies of Mycobacterium avium subspecies hominissuis in lymph nodes sampled at slaughter from pigs of different weeks of age

\begin{tabular}{lccccccc}
\hline & \multicolumn{2}{c}{ Mandibular lymph nodes } & & \multicolumn{2}{c}{ Jejunal lymph nodes } \\
\cline { 2 - 3 } & $\begin{array}{c}\text { Samples } \\
\text { examined }\end{array}$ & \multicolumn{2}{c}{$\begin{array}{c}\text { Positive } \\
\text { samples }\end{array}$} & & $\begin{array}{c}\text { Samples } \\
\text { examined }\end{array}$ & & $\begin{array}{c}\text { Positive } \\
\text { samples }\end{array}$ \\
\hline Weeks & $\mathbf{n}$ & $\mathbf{n}$ & $\mathbf{\%}$ & & $\mathbf{n}$ & $\mathbf{n}$ & $\mathbf{\%}$ \\
$\mathbf{2 4}$ & 38 & 23 & $\mathbf{6 0}$ & & 33 & 11 & $\mathbf{3 3}$ \\
$\mathbf{2 5}$ & 45 & 28 & $\mathbf{6 2}$ & & 52 & 21 & $\mathbf{4 0}$ \\
$\mathbf{2 6}$ & 15 & 10 & $\mathbf{6 7}$ & & 13 & 7 & $\mathbf{5 4}$ \\
$\mathbf{2 8}$ & 25 & 20 & $\mathbf{8 0}$ & & 29 & 21 & $\mathbf{7 2}$ \\
\hline
\end{tabular}

Grossly detectable changes, such as abnormal consistency, colour or macroscopically visible changes, were recorded in 38 (31\%) of the mandibular and $27(21 \%)$ of the jejunal lymph nodes sampled. Mycobacterium avium subsp. hominissuis was detected in a large number of lymph nodes without lesions, and Table 4 shows the relationship between the presence of grossly detectable changes and the presence of live M. avium subsp. hominissuis.

\section{Investigation of relatedness between $M$. avium isolates}

All isolates from peat $(n=16)$ and faeces $(n=11)$, and a random selection of 47 (33\%) of the 141 isolates from lymph nodes, underwent MLVA analysis. MLVA analysis identified 13 different profiles among the 74 analysed isolates, distributed on seven clusters and six singletons (Figure 1). Clusters were recognised when containing $\geq 2$ isolates with identical profile. The present analysis showed clusters of different sizes, ranging from two to 28 isolates. In two samples from peat, two different MLVA profiles were detected within the same sample. In the other nine peat and all 11 faecal samples, isolates from the same sample showed identical MLVA profiles, and only one representative from each sample is shown in the dendrogram. Multiple MLVA profiles were detected in all types of sample material, and isolates from peat, faeces and lymph nodes clustered together on several occasions. However, due to the animals not being individually numbered, further investigation of the potential presence of multiple profiles within the same animal was not possible to perform.

\section{Discussion}

The presence of M. avium subsp. hominissuis in faeces in naturally infected pigs was demonstrated in the present study. Together with the similar finding in our recent study with experimentally infected pigs [21], the suggestion of faeces playing a potential role in the spread of $M$. avium subsp. hominissuis infection is strengthened. Our findings are in concordance with older literature on studies of M. avium in pigs [35-37], but to our best knowledge, the present study is the first of its kind 
Table 4 Detection frequencies of Mycobacterium avium subspecies hominissuis in mandibular and jejunal lymph nodes with and without gross pathological lesions from pigs slaughtered at different age

\begin{tabular}{|c|c|c|c|c|c|}
\hline \multirow{2}{*}{$\begin{array}{l}\text { Lymph node } \\
\text { Mandibular }\end{array}$} & \multirow{2}{*}{ Weeks } & \multicolumn{2}{|c|}{$\begin{array}{c}\text { Pathological changes } \\
\text { present }\end{array}$} & \multicolumn{2}{|c|}{$\begin{array}{c}\text { Pathological changes } \\
\text { absent }\end{array}$} \\
\hline & & $\begin{array}{c}\text { Total } \\
\text { number }\end{array}$ & $\begin{array}{l}\text { Culture } \\
\text { positive }\end{array}$ & $\begin{array}{c}\text { Total } \\
\text { number }\end{array}$ & $\begin{array}{l}\text { Culture } \\
\text { positive }\end{array}$ \\
\hline & 24 & 8 & $100 \%$ & 30 & $53 \%$ \\
\hline & 25 & 15 & $93 \%$ & 30 & $43 \%$ \\
\hline & 26 & 6 & $100 \%$ & 9 & $44 \%$ \\
\hline & 28 & 10 & $90 \%$ & 15 & $73 \%$ \\
\hline & Total & 39 & $95 \%$ & 84 & $52 \%$ \\
\hline \multirow[t]{7}{*}{ Jejunal } & Weeks & \multicolumn{2}{|c|}{$\begin{array}{c}\text { Pathological changes } \\
\text { present }\end{array}$} & \multicolumn{2}{|c|}{$\begin{array}{c}\text { Pathological changes } \\
\text { absent }\end{array}$} \\
\hline & & $\begin{array}{c}\text { Total } \\
\text { number }\end{array}$ & $\begin{array}{l}\text { Culture } \\
\text { positive }\end{array}$ & $\begin{array}{c}\text { Total } \\
\text { number }\end{array}$ & $\begin{array}{l}\text { Culture } \\
\text { positive }\end{array}$ \\
\hline & 24 & 3 & $100 \%$ & 30 & $27 \%$ \\
\hline & 25 & 6 & $100 \%$ & 46 & $33 \%$ \\
\hline & 26 & 3 & $100 \%$ & 10 & $40 \%$ \\
\hline & 28 & 15 & $100 \%$ & 14 & $43 \%$ \\
\hline & Total & 27 & $100 \%$ & 109 & $42 \%$ \\
\hline
\end{tabular}

to methodically study faecal excretion of $M$. avium subsp. hominissuis in naturally infected pigs. Additionally, the detection of identical isolates in peat, faeces and lymph nodes, suggests that peat was the primary source of infection for the herd. This assumption is supported by previous research describing a link between $M$. avium infections in swine and peat $[20,27]$. Mycobacterium avium subsp. hominissuis in faeces might originate merely from peat passing through the alimentary tract, but the absence of $M$. avium subsp. hominissuis in faeces from the sows in the study, who also were kept on peat, support a theory of M. avium subsp. hominissuis being excreted from jejunal lesions. In our previous study of experimental infection of piglets at an early age with $M$. avium subsp. hominissuis and M. avium subsp. avium, M. avium subsp. hominissuis was detected in faeces at six weeks, which reinforces the conclusion that the bacterium was not just passing through the digestive system [21]. The frequent detection of M. avium subsp. hominissuis in jejunal lymph nodes is also suggestive of a link between intestinal affection and the presence of $M$. avium subsp. hominissuis in faeces. A theory of transmission of $M$. avium subsp. hominissuis between animals by the faecal-oral route was not rejected by the present MLVA results, regarding the fact that isolates from peat, faeces and lymph nodes clustered together frequently. Faecal excretion of $M$. avium subsp. hominissuis might contribute to enhanced pressure of infection in a herd and maintain the possibility of transmission between animals. However, it is not possible to compare the role of faeces and peat in infection of pigs based on the present results.

Mycobacterium avium subsp. hominissuis was frequently detected in mandibular and jejunal lymph nodes of slaughtered pigs from the breeding facility that used peat, and meat from this herd was used for human consumption without any intervention. Importantly, on several occasions $M$. avium subsp. hominissuis was cultured from lymph nodes without any macroscopically visible changes indicative of infection, in concordance with our previous study of experimental $M$. avium infection in pigs [21]. The great extent of M. avium subsp. hominissuis present within lymph nodes claims for further knowledge about the presence of the bacterium in the remaining porcine organism. The findings suggest that lesions might very well not be visible to the naked eye, and that $M$. avium subsp. hominissuis is very likely to be found in carcasses from pigs raised on peat. Similar observations have also been described previously [20,27]. Further investigations could be important in order to secure the health of people handling pork carcasses and meat, and of the consumers. Reports on M. avium subsp. hominissuis isolates from pigs and humans being closely related $[2,4,22-25]$, together with the fact that a common source of $M$. avium subsp. hominissuis infection in these two mammalian species is yet to be identified, strengthen the theory generated by the present study that porcine intestinal organs or pork meat can be a potential source of human M. avium subsp. hominissuis infection. Other authors have reported detection of $M$. avium subsp. hominissuis DNA in muscle tissue of pigs artificially infected with $M$. avium subsp. hominissuis $[18,19]$. The question that remains is whether M. avium subsp. hominissuis could be present outside of lymph nodes in unapparent porcine carcasses. Further investigations, aiming at thoroughly mapping the presence of $M$. avium subsp. hominissuis in muscles, lungs and livers of slaughtered pigs, would be required to secure an up-to-date mode of animal husbandry and meat inspection and thereby protect human health. Depending on the outcome of such future investigations, the need to apply novel techniques at meat control to rapidly identify the presence of M. avium in porcine tissues [38] might be appropriate to consider.

In the present study, a variety of other NTM of significance for human health were isolated from peat, faeces and lymph nodes. Although no further attempt was done to investigate the relatedness between isolates to elucidate routes of transmission, the findings could provide helpful information about possible sources for both porcine and human infection. Species isolated from all the three types of sample material were $M$. bohemicum, $M$. celatum and M. palustre, of which all have been 
reported to have relevance for human health. Both $M$. bohemicum and $M$. palustre have been described as causative agents of peadiatric lymphadenitis [39-42] and should be regarded as potential pathogens [6]. Mycobacterium celatum can cause disseminated infection or lung infection in humans with underlying disease [6], paediatric 
lymphadenitis [43] and serious pulmonary infection in apparently immunocompetent individuals $[44,45]$. Isolation of $M$. celatum from porcine lymph nodes has previously been reported only once [46]. Mycobacterium malmoense was found only in peat and faeces in the present study, but has been detected in porcine lymph nodes by others [5]. It is increasingly recognised as an important lung pathogen in humans [47], but has also been associated with human lymphadenitis and septic arthritis [48,49]. Mycobacterium branderi, reported to cause human lung disease and ulcerative tenosynovitis [50,51], was on one occasion detected in faeces in the present study. In partially concordance with our present findings, van Ingen et al. [5] cultured M. malmoense, M. bohemicum and $M$. palustre from porcine lymph nodes. Our supplementary isolation of the same species in peat, together with the absence of NTM in lymph nodes from pigs not raised on peat bedding, suggests peat as a source of infection in pigs. Although the identified NTM species already are considered to be environmental, enrichment in peat and faeces might be of relevance for establishment of infection in pigs, with the further possibility of transmission to humans.

To conclude, faeces might be of importance for transmission of $M$. avium subsp. hominissuis between animals and for maintenance of the infection pressure in pig herds. Also, pigs might be heavily infected with $M$. avium subsp. hominissuis, despite their carcasses being macroscopically unapparent with regards to mycobacterial presence. Peat represents an important source of M. avium subsp. hominissuis, but also of other NTM that might be of increasing significance for porcine and human health. All in all, these findings should stimulate further investigation of the presence of mycobacteria in pig carcasses and reconsiderations with regards to animal husbandry, as well as to the current practice of meat control.

\section{Additional file}

\section{Additional file 1: Primers for MLVA analysis and $16 \mathrm{~s}$ rDNA}

sequencing. Table describing the primers used for the eight locus MLVA analysis and for the 165 rDNA sequencing analysis.

\section{Competing interests}

The authors declare that they have no competing interests.

\section{Authors' contributions}

AA was responsible for conception and design of the experiment, performed sampling, laboratory work, data analysis, and drafted the manuscript. 10 contributed to conception and design of the experiment, sampling, and to critical revision of the manuscript. AJ was involved in conception and design of the experiment, sampling and in critical revision of the manuscript. BD participated in conception and design of the experiment, laboratory work and in critical revision of the manuscript. TBJ contributed to conception and design of the experiment, sampling, laboratory work, MLVA data analysis and helped to draft the manuscript. All authors have read and approved the final manuscript.

\section{Acknowledgements}

The project was funded by the Research Council of Norway and the Norwegian Veterinary Institute (NVI). Cathrine Hexeberg at The Norwegian Pig Health Service, Animalia, is acknowledged for her excellent assistance in communication with the involved swine producers and faecal sampling. The staff at The Norwegian Food Safety Authority, District Office Ytre Østfold, is greatly acknowledged for their support during planning and performance of sample collection. Bjørn Lium at the NVI is acknowledged for sharing his expertise and for excellent consultation, and Nina Fundingsrud and Sigrun Nilsen at the NVI are greatly acknowledged for performing laboratory analysis. The owners of Herd A and Herd B also deserve credit for providing access to their facilities and for contributing to handling of the animals during sampling.

\section{Author details}

${ }^{1}$ Norwegian Veterinary Institute, P.O. Box 750 Sentrum, N-0106 Oslo, Norway. ${ }^{2}$ Norwegian Pig Health Service, Animalia, P.O. Box 396 Økern, N-0513 Oslo, Norway.

Received: 20 January 2014 Accepted: 1 April 2014

Published: 17 April 2014

\section{References}

1. Inderlied CB, Kemper CA, Bermudez LE: The Mycobacterium avium complex. Clin Microbiol Rev 1993, 6:266-310

2. Mijs W, de Haas P, Rossau R, Van der Laan T, Rigouts L, Portaels F, Van Soolingen D: Molecular evidence to support a proposal to reserve the designation Mycobacterium avium subsp. avium for bird-type isolates and ' $M$. avium subsp. hominissuis' for the human/porcine type of $M$. avium. Int J Syst Evol Microbiol 2002, 52:1505-1518.

3. Tell LA, Woods L, Cromie RL: Mycobacteriosis in birds. Rev Sci Tech 2001, 20:180-203

4. Johansen TB, Olsen I, Jensen MR, Dahle UR, Holstad G, Djonne B: New probes used for IS1245 and IS1311 restriction fragment length polymorphism of Mycobacterium avium subsp. avium and Mycobacterium avium subsp. hominissuis isolates of human and animal origin in Norway. BMC Microbiol 2007, 7:14

5. van Ingen J, Wisselink HJ, van Solt-Smits CB, Boeree MJ, Van Soolingen D: Isolation of mycobacteria other than Mycobacterium avium from porcine lymph nodes. Vet Microbiol 2010, 144:250-253.

6. Tortoli E: Impact of genotypic studies on mycobacterial taxonomy: the new mycobacteria of the 1990s. Clin Microbiol Rev 2003, 16:319-354.

7. Jarzembowski JA, Young MB: Nontuberculous mycobacterial infections. Arch Pathol Lab Med 2008, 132:1333-1341.

8. Eisenberg T, Volmer R, Eskens U, Moser I, Nesseler A, Sauerwald C, Seeger H, Klewer-Fromentin K, Mobius P: Outbreak of reproductive disorders and mycobacteriosis in swine associated with a single strain of Mycobacterium avium subspecies hominissuis. Vet Microbiol 2012, 159:69-76.

9. Wellenberg GJ, de Haas PE, van Ingen J, Van Soolingen D, Visser IJ: Multiple strains of Mycobacterium avium subspecies hominissuis infections associated with aborted fetuses and wasting in pigs. Vet Rec 2010, 167:451-454.

10. Thorel MF, Huchzermeyer $\mathrm{H}$, Weiss R, Fontaine JJ: Mycobacterium avium infections in animals. Literature review. Vet Res 1997, 28:439-447.

11. Domingos M, Amado A, Botelho A: IS1245 RFLP analysis of strains of Mycobacterium avium subspecies hominissuis isolated from pigs with tuberculosis lymphadenitis in Portugal. Vet Rec 2009, 164:116-120.

12. Hibiya K, Utsunomiya K, Yoshida T, Toma S, Higa F, Tateyama M, Fujita J: Pathogenesis of systemic Mycobacterium avium infection in pigs through histological analysis of hepatic lesions. Can J Vet Res 2010, 74:252-257.

13. Biet F, Boschiroli ML, Thorel MF, Guilloteau LA: Zoonotic aspects of Mycobacterium bovis and Mycobacterium avium-intracellulare complex (MAC). Vet Res 2005, 36:411-436.

14. Falkinham JO 3rd, Iseman MD, de Haas P, van Soolingen D: Mycobacterium avium in a shower linked to pulmonary disease. J Water Health 2008 6:209-213.

15. Falkinham JO 3rd: Nontuberculous mycobacteria from household plumbing of patients with nontuberculous mycobacteria disease. Emerg Infect Dis 2011, 17:419-424

16. Lumb R, Stapledon R, Scroop A, Bond P, Cunliffe D, Goodwin A, Doyle R, Bastian I: Investigation of spa pools associated with lung disorders 
caused by Mycobacterium avium complex in immunocompetent adults. Appl Environ Microbiol 2004, 70:4906-4910.

17. Ichiyama S, Shimokata K, Tsukamura M: The isolation of Mycobacterium avium complex from soil, water, and dusts. Microbiol Immunol 1988, 32:733-739

18. Klanicova B, Slana I, Vondruskova H, Kaevska M, Pavlik I: Real-time quantitative PCR detection of Mycobacterium avium subspecies in meat products. J Food Prot 2011, 74:636-640.

19. Slana I, Kaevska M, Kralik P, Horvathova A, Pavlik I: Distribution of Mycobacterium avium subsp. avium and M. a. hominissuis in artificially infected pigs studied by culture and IS901 and IS1245 quantitative real time PCR. Vet Microbiol 2010, 144:437-443.

20. Agdestein A, Johansen TB, Polacek V, Lium B, Holstad G, Vidanovic D, Aleksic-Kovacevic S, Jorgensen A, Zultauskas J, Nilsen SF, Djønne B: Investigation of an outbreak of mycobacteriosis in pigs. BMC Vet Res 2011, 7:63.

21. Agdestein A, Johansen TB, Kolbjornsen O, Jorgensen A, Djonne B, Olsen I: A comparative study of Mycobacterium avium subsp. avium and Mycobacterium avium subsp. hominissuis in experimentally infected pigs. BMC Vet Res 2012, 8:11.

22. Komijn RE, de Haas PE, Schneider MM, Eger T, Nieuwenhuijs JH, van den Hoek RJ, Bakker D, van Zijd Erveld FG, van Soolingen D: Prevalence of Mycobacterium avium in slaughter pigs in The Netherlands and comparison of IS1245 restriction fragment length polymorphism patterns of porcine and human isolates. J Clin Microbiol 1999, 37:1254-1259.

23. Radomski N, Thibault VC, Karoui C, de Cruz K, Cochard T, Gutierrez C, Supply $P$, Biet F, Boschiroli ML: Determination of genotypic diversity of Mycobacterium avium subspecies from human and animal origins by mycobacterial interspersed repetitive-unit-variable-number tandem-repeat and IS1311 restriction fragment length polymorphism typing methods. J Clin Microbiol 2010, 48:1026-1034.

24. Tirkkonen T, Pakarinen J, Moisander AM, Makinen J, Soini H, Ali-Vehmas T: High genetic relatedness among Mycobacterium avium strains isolated from pigs and humans revealed by comparative IS1245 RFLP analysis. Vet Microbiol 2007, 125:175-181.

25. Tirkkonen T, Pakarinen J, Rintala E, Ali-Vehmas T, Marttila H, Peltoniemi OA, Makinen J: Comparison of variable-number tandem-repeat markers typing and IS1245 restriction fragment length polymorphism fingerprinting of Mycobacterium avium subsp. hominissuis from human and porcine origins. Acta Vet Scand 2010, 52:21.

26. Matlova L, Dvorska L, Palecek K, Maurenc L, Bartos M, Pavlik I: Impact of sawdust and wood shavings in bedding on pig tuberculous lesions in lymph nodes, and IS1245 RFLP analysis of Mycobacterium avium subsp. hominissuis of serotypes 6 and 8 isolated from pigs and environment. Vet Microbiol 2004, 102:227-236.

27. Matlova L, Dvorska L, Ayele WY, Bartos M, Amemori T, Pavlik I: Distribution of Mycobacterium avium complex isolates in tissue samples of pigs fed peat naturally contaminated with mycobacteria as a supplement. J Clin Microbiol 2005, 43:1261-1268.

28. Fischer OA, Matlova L, Bartl J, Dvorska L, Svastova P, du Maine R, Melicharek I, Bartos M, Pavlik I: Earthworms (Oligochaeta, Lumbricidae) and mycobacteria. Vet Microbiol 2003, 91:325-338.

29. Saxegaard F: Isolation of Mycobacterium paratuberculosis from intestinal mucosa and mesenteric lymph nodes of goats by use of selective Dubos medium. J Clin Microbiol 1985, 22:312-313.

30. Ahrens P, Giese SB, Klausen J, Inglis NF: Two markers, IS901-IS902 and p40, identified by PCR and by using monoclonal antibodies in Mycobacterium avium strains. J Clin Microbiol 1995, 33:1049-1053.

31. Johansen TB, Djonne B, Jensen MR, Olsen I: Distribution of IS1311 and IS1245 in Mycobacterium avium subspecies revisited. J Clin Microbiol 2005, 43:2500-2502.

32. Kankya C, Muwonge A, Djonne B, Munyeme M, Opuda-Asibo J, Skjerve E, Oloya J, Edvardsen V, Johansen TB: Isolation of non-tuberculous mycobacteria from pastoral ecosystems of Uganda: public health significance. BMC Public Health 2011, 11:320

33. Basic local alignment search tool. [http://blast.ncbi.nlm.nih.gov/Blast.cgi].

34. Thibault VC, Grayon M, Boschiroli ML, Hubbans C, Overduin P, Stevenson K, Gutierrez MC, Supply P, Biet F: New variable-number tandem-repeat markers for typing Mycobacterium avium subsp. paratuberculosis and $M$. avium strains: comparison with IS900 and IS1245 restriction fragment length polymorphism typing. J Clin Microbiol 2007, 45:2404-2410.
35. Jorgensen JB: Experimental infection with Mycobacterium avium, serotype 2 , in pigs. 4 . Contact infection from orally inoculated pigs. Acta Vet Scand 1978, 19:58-72.

36. Thoen CO, Johnson DW, Himes EM, Menke SB, Muscoplat CC: Experimentally induced Mycobacterium avium serotype 8 infection in swine. Am J Vet Res 1976, 37:177-181.

37. Ellsworth S, Kirkbride CA, Johnson DD: Excretion of Mycobacterium avium from lesions in the intestine and tonsils of infected swine. Am J Vet Res 1980, 41:1526-1530.

38. Tirkkonen T, Nieminen T, Ali-Vehmas T, Peltoniemi OA, Wellenberg GJ, Pakarinen J: Quantification of Mycobacterium avium subspecies in pig tissues by real-time quantitative PCR. Acta Vet Scand 2013, 55:26.

39. Tortoli E, Bartoloni A, Manfrin V, Mantella A, Scarparo C, Bottger E: Cervical lymphadenitis due to Mycobacterium bohemicum. Clin Infect Dis 2000, 30:210-211.

40. Huber J, Richter E, Binder L, Maass M, Eberl R, Zenz W: Mycobacterium bohemicum and cervical lymphadenitis in children. Emerg Infect Dis 2008, 14:1158-1159.

41. Torkko P, Suomalainen S, livanainen E, Suutari M, Paulin L, Rudback E, Tortoli E, Vincent V, Mattila R, Katila ML: Characterization of Mycobacterium bohemicum isolated from human, veterinary, and environmental sources. J Clin Microbiol 2001, 39:207-211.

42. Torkko P, Suomalainen S, livanainen E, Tortoli E, Suutari M, Seppanen J, Paulin L, Katila ML: Mycobacterium palustre sp. nov., a potentially pathogenic, slowly growing mycobacterium isolated from clinical and veterinary specimens and from Finnish stream waters. Int I Syst Evol Microbiol 2002, 52:1519-1525.

43. Haase G, Skopnik H, Batge S, Bottger EC: Cervical lymphadenitis caused by Mycobacterium celatum. Lancet 1994, 344:1020-1021

44. Bux-Gewehr I, Hagen HP, Rusch-Gerdes S, Feurle GE: Fatal pulmonary infection with Mycobacterium celatum in an apparently immunocompetent patient. J Clin Microbiol 1998, 36:587-588,

45. Piersimoni C, Tortoli E, de Lalla F, Nista D, Donato D, Bornigia S, de Sio G: Isolation of Mycobacterium celatum from patients infected with human immunodeficiency virus. Clin Infect Dis 1997, 24:144-147.

46. Pate M, Kusar D, Zolnir-Dove M, Ocepek M: MIRU-VNTR typing of Mycobacterium avium in animals and humans: Heterogeneity of Mycobacterium avium subsp. hominissuis versus homogeneity of Mycobacterium avium subsp. avium strains. Res Vet Sci 2011, 91:376-381.

47. Moore JE, Kruijshaar ME, Ormerod LP, Drobniewski F, Abubakar I: Increasing reports of non-tuberculous mycobacteria in England, Wales and Northern Ireland, 1995-2006. BMC Public Health 2010, 10:612.

48. Hoefsloot W, Boeree MJ, van Ingen J, Bendien S, Magis C, de Lange W, Dekhuijzen PN, van Soolingen D: The rising incidence and clinical relevance of Mycobacterium malmoense: a review of the literature. Int J Tuberc Lung Dis 2008, 12:987-993.

49. Talbot CL, Rhodes B: An atypical mycobacterial infection of the shoulder. Int J Shoulder Surg 2012, 6:64-66.

50. Koukila-Kahkola P, Springer B, Bottger EC, Paulin L, Jantzen E, Katila ML: Mycobacterium branderi sp. nov., a new potential human pathogen. Int J Syst Bacteriol 1995, 45:549-553.

51. Wolfe J, Turenne C, Alfa M, Harding G, Thibert L, Kabani A: Mycobacterium branderi from both a hand infection and a case of pulmonary disease. J Clin Microbiol 2000, 38:3896-3899.

doi:10.1186/1297-9716-45-46

Cite this article as: Agdestein et al:: Novel insights into transmission routes of Mycobacterium avium in pigs and possible implications for human health. Veterinary Research 2014 45:46. 